\title{
The use of a Fogarty Catheter in a Case of an Endobronchial Blood Clot in a Laryngectomy Patient
}

Billy Wong

\section{ABSTRACT}

Introduction: Endobronchial blood clot causing airway obstruction is a rare but potentially life-threatening condition and can occur in a variety of clinical settings.

Case report: A male laryngectomy, with a background of bronchogenic carcinoma was treated conservatively for tracheitis following an episode of upper respiratory tract infection. However, he later developed airway obstruction secondary to endobronchial bleeding with endobronchial clot formation. Conventional methods of suctioning and rigid bronchoscopy with forceps failed to remove the hematoma. A Fogarty embolectomy catheter was used to remove the hematoma, relieving the airway obstruction.

Discussion: The Fogarty embolectomy catheter has been widely used in various vascular operations for removal of arterial and venous emboli over the last years. However, its application in nonvascular cases is not widely published.

Conclusion: We report a case of tracheitis, complicated by an endobronchial clot in a laryngectomy patient, and demonstrated the use of Fogarty embolectomy catheter in such case when conventional methods fail.

Keywords: Blood clot, Endobronchial, Fogarty, Laryngectomy.

How to cite this article: Wong $B$. The use of a Fogarty Catheter in a Case of an Endobronchial Blood Clot in a Laryngectomy Patient. Int J Head Neck Surg 2015;6(3):118-120.

\section{Source of support: Nil}

Conflict of interest: None

\section{INTRODUCTION}

Endobronchial blood clot causing airway obstruction is a rare but potentially life-threatening condition and can occur in a variety of clinical settings. According to the literature, the reported causes of endobronchial blood clot include bronchiectasis, tuberculosis, mitral stenosis, pulmonary infection, pulmonary ateriovenous malformation, sarcoidosis, bronchial carcinoma, intrathoracic trauma, and mucosal trauma from catheter manipulation,

\section{Trainee}

Department of Otolaryngology, Addenbrooke's Hospital Cambridge, United Kingdom

Corresponding Author: Billy Wong, Trainee, Department of Otolaryngology, Addenbrooke's Hospital, Cambridge, United Kingdom, e-mail: bwonglk@gmail.com bronchoalveolar lavage, transbronchial biopsy and tracheostomy placement. ${ }^{1-4}$ Many endobronchial bleeding are preceeded by hemoptysis but this can be absent especially in patients who had prolonged mechanical ventilation or tracheostomy placement. ${ }^{4}$ Hence, the diagnosis is established by direct endoscopic evaluation of the clot.

Various methods of clot removal including bronchoscopy with suctioning and forceps extraction, thrombolysis and cryotherapy have been described in the literature, all with reasonable success rates. The exact method used of course depends on the staff and equipment available at the time, as well as the experience of the attending anesthetist and surgeon. However, one technique which is relatively underused in this scenario is the Fogarty embolectomy catheter.

We report a case of endobronchial bleeding which has caused an organized clot to form in the distal trachea and left main bronchus, and subsequently described our method of clot removal together with a review of the literature.

\section{CASE REPORT}

A 74-year-old Caucasian gentleman, who underwent total laryngectomy and postoperative radiotherapy for squamous cell carcinoma of the larynx 13 years ago, presented to the accident and emergency department with mild bleeding and crusting around the stoma site.

On examination, there were no signs of stridor and the patient maintained normal respiration on room air. There appeared to be some crusting and dry blood around the stoma site which was carefully removed with forceps. Flexible tracheoscopy via the stoma was also performed which showed moderate degree of crusting and edema of the tracheal mucosa but there was no active bleeding and the airway was otherwise clear down to the level of the carina. The patient was nonetheless admitted and treated for tracheitis secondary to lower respiratory tract infection.

However, he began to develop stridor with persistent oxygen desaturation the next morning. He was found to have streaks of blood around the stoma site. Suctioning of the trachea with bronchial suction catheter was attempted but his condition remained unchanged. A repeat flexible tracheoscopy examination via the stoma 
identified an organized clot in the distal trachea and left main bronchus, which had formed a perfect 'cast' of the airway, causing a subtotal airway obstruction.

He was transferred to the intensive care unit (ICU) and his oxygen saturation was maintained at around 85 to $90 \%$ with oxygen provided via a stomal mask with positive pressure ventilation from a reservoir bag.

An ENT/Head and Neck consultant surgeon attended to the patient in ICU and carried out a rigid bronchoscopy via the stoma. Removal of the clot was attempted with forceps on several occasions without success. The semisolid clot had a consistency that was too friable for the forceps to obtain a purchase, and yet too congealed to be suctioned out by bronchial suction catheter.

A Fogarty embolectomy catheter was then used to attempt removal of the clot. The catheter was inserted under flexible tracheoscope guidance. The tip of the catheter was advanced 5 to $6 \mathrm{~cm}$ distal to the clot. The balloon was then inflated with $2 \mathrm{ml}$ of saline and the catheter withdrawn slowly in a retrograde fashion.

A $4.5 \mathrm{~cm}$-long-organized clot was extracted on the second attempt (Fig. 1). The airway obstruction was relieved and the patient's condition stabilized. Subsequent tracheobronchoscopy revealed no residual abnormality. Ventilation was effective without further incident. Regular suction and humidified oxygen were resumed and there were no further problems.

\section{DISCUSSION}

To the best of our knowledge, 43 cases of endobronchial blood clot have been reported in the literature to date and many different techniques have been employed successfully in removing the airway clots, but the use of Fogarty embolectomy catheter is still understated. ${ }^{1-4}$

Collins and Presnell described two cases in 2005, where the diagnosis were only confirmed on postmortem.

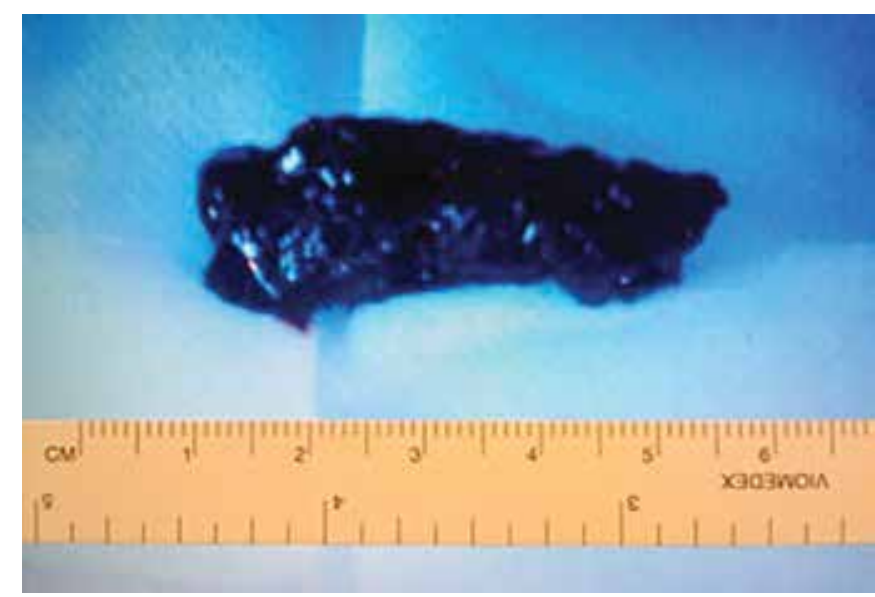

Fig. 1: Endobronchial blood clot which was removed with Fogarty catheter
Two anesthetic/critical care medicine papers have described successful removal of endobronchial clots by way of 'whole tube suction' (i.e. applying complete suction to the endotracheal tubes) in 2002 and 2004. ${ }^{2,3}$ Botnick et al $(1994)^{5}$ and Vajo et al (1996) ${ }^{6}$ reported the use of topical urokinase and streptokinase respectively for endobronchial thrombolysis.

Arney et al (1999) ${ }^{4}$ described three cases of airway obstruction by blood clot and carried out a comprehensive literature review. They reported methods that have been utilized in previous reports including suctioning, ${ }^{4}$ forceps extraction with flexible ${ }^{4} /$ rigid bronchoscope $^{10}$ topical thrombolytic agents ${ }^{5,6}$ and lastly Fogarty catheter.

Weerdt et al (2005) successfully removed a large endobronchial blood clot in a patient with massive hemoptysis using cryotherapy. They described this technique as the treatment of choice for removal of large clots from airways because of its freezing characteristics which enabled the removal of water containing foreign bodies. ${ }^{10}$

Allen and Siefkin (1987) are the only authors to describe the use of a Fogarty catheter in the treatment of endobronchial blood clot in 3 patients. ${ }^{7}$ Fitzwater et al (2008) recently described the use of a Fogarty catheter under tracheoscopy guidance in successfully removing tracheal 'thrombi' in a 7-year-old Spaniel-crossbreed dog. ${ }^{8}$

The Fogarty embolectomy catheter has been widely used in various vascular operations for removal of arterial and venous emboli over the last 30 to 40 years. However, its application in non-vascular cases is not widely published and to our knowledge, this is the only 4th case describing its use in this clinical setting in the English literature.

Utilization of a Fogarty catheter in the case of an airway clot offers many advantages. As demonstrated in this case, the consistency of blood clot may sometimes prove challenging for conventional methods, such as suctioning or forceps, but an alternative technique offered by the use of Fogarty catheter may make removing a stubborn clot in the airway may rather straight forward.

The smaller size of the catheter compared to other removal methods also confers another plus point as this could avoid further trauma to the damaged tracheobronchial mucosa. Although we used the Fogarty catheter under the guidance of a traceheoscope, the fact that the catheter is also radiopaque can aid visualization in difficult cases.

\section{CONCLUSION}

We have reported a case of tracheitis, complicated by upper airway obstruction by an endobronchial clot in a laryngectomy patient. Utilization of Fogarty embolectomy catheter had enabled safe clot removal with restoration of 
adequate ventilation. This was made easier by the patient having a laryngostome due to prior total laryngectomy. In our limited experience, the Fogarty catheter has been effective in removal of the endobronchial clot, and its use should be, therefore, be considered when other conventional methods fail.

\section{REFERENCES}

1. Collins KA, Presnell SE. Asphyxia by tracheobronchial thrombus. Am J Forensic Med Pathol 2005;26(4):327-329.

2. Bodenham AR. Removal of obstructing blood clot from the lower airway: an alternative suction technique. Anaesthesia 2002;57(1):40-43.

3. Dongelmans DA, Jonkers RE, Schultz MJ. Case report: a ball valve blood clot in the airways-life-saving whole tube suction. Crit Care 2004;8(5):R289-290.

4. Arney KL, Judson MA, Sahn SA. Airway obstruction arising from blood clot: three reports and a review of the literature. Chest 1999;115(1):293-300.
5. Botnick W, Brown H. Endobronchial urokinase for dissolution of massive clot following transbronchial biopsy. Chest 1994;105(3):953-954.

6. Vajo Z, Parish JM. Endobronchial thrombolysis with streptokinase for airway obstruction due to blood clots. Mayo Clin Proc 1996;71(6):595-596.

7. Allen RP, Siefkin AD. Emergency airway clot removal in acute hemorrhagic repiratory failure. Crit Care Med 1987;15(10): 985-986.

8. Fitzwater KL, Marks SL, Hanel RM. Thrombi in the trachea of a dog secondary to placement of a tracheotomy tube. J Am Vet Med Assoc 2008;233(5):758-760.

9. Sprug J, Bourke DL, Harrison C, Barnas GM. Endotracheal tube and tracheobronchial obstruction as causes of hypoventilation with high inspiratory pressures. Chest 1994;105(2): 550-552.

10. Weerdt SD, Noppen M, Remels L, Vanherreweghe R, Meysman M, Vincken W. Successful removal of a massive endobronchial blood clot by means of cryotherapy. J Bronchol Dedicat Bronchos Interven Pulmonol 2005;12(1):23-24. 